\title{
NLOS Signal Detection Based on Single Orthogonal Dual-Polarized GNSS Antenna
}

\author{
Ke Zhang, Baiyu Li, Xiangwei Zhu, Huaming Chen, and Guangfu Sun \\ College of Electronic Science and Engineering, National University of Defense Technology, Changsha 410073, China \\ Correspondence should be addressed to Xiangwei Zhu; zhuxiangwei@nudt.edu.cn
}

Received 30 December 2016; Accepted 2 March 2017; Published 13 April 2017

Academic Editor: Miguel Ferrando Bataller

Copyright (C) 2017 Ke Zhang et al. This is an open access article distributed under the Creative Commons Attribution License, which permits unrestricted use, distribution, and reproduction in any medium, provided the original work is properly cited.

\begin{abstract}
Nowadays users have a high demand for the accuracy of position and velocity, but errors caused by non-line-of-sight (NLOS) signals cannot be removed effectively. Since the GNSS signal is right-hand circular polarized (RHCP), the axial ratio of the strong NLOS signal is larger than that of the Line-of-Sight (LOS) signal. Based on the difference of the axial ratio, a method for NLOS signal detection using single orthogonal dual-polarized antenna is proposed. The antenna has two channels to receive two orthogonal linear polarized components of the incoming signals. Parallel cross-cancellation is used to remove the LOS signal while maintaining most of the NLOS signals from the receiving signals. The residual NLOS signals are then detected by conventional GNSS digital processor in real time without any prior knowledge of their characteristics. The proposed method makes use of the polarization and spatial information and can detect long delay NLOS signal by miniature and inexpensive receiver GNSS. The effectiveness of the proposed method is confirmed by simulation data.
\end{abstract}

\section{Introduction}

In the urban canyon and valley, buildings and other obstacles may block the LOS signal of many satellites. NLOS signals represent the signals where the direct signals are blocked and signals are received only via reflections. Reception of these reflected signals results in significant positioning errors. NLOS signal reception results in a pseudorange measurement error equal to the additional path delay taken by the reflected signal versus the direct path between satellite and receiver. So NLOS signal detection and mitigation are urgently needed in these receivers when they are used in the urban canyon and valley.

NLOS signal and multipath interference have some reflected characteristics in common when reflected by buildings or other obstacles. Signal processing techniques such as narrow correlation and double-delta multiple correlators [1] can be used to mitigate multipath. In multiple-antenna receivers, adaptive beamforming can be applied to remove the LOS signal from the receiving signals [2]. Then the multipath can be detected from residual signal by the regular delay lock loop (DLL). Furthermore adaptive multipath mitigation algorithms have been proposed to null the multipath using nonuniform antenna array, such as moving array [3], switch array [4], and vector array [5]. Though these algorithms could effectively mitigate the effect of multipath with the previous knowledge of LOS signal, they are of little use for mitigating NLOS signal since LOS signal is blocked. NLOS signal detection using a standard receiver requires signals from different satellites. Consistency-checking techniques [6] can identify and eliminate NLOS signals when most of the other received signals are LOS signal with minimal multipath interference. The marginalized likelihood ratio test (MLRT) is used to detect, identify, and estimate the corresponding NLOS signal [7]. The NLOS signal can be detected and mitigated by judging measurement of more satellites using Receiver Autonomous Integrity Monitoring (RAIM) [8]. These techniques are less reliable and useless when the number of satellites in view is limited. Polarized diversity makes the multiplepolarized antenna receiver able to distinguish different sources [9]. RHCP and left-hand circular polarized (LHCP) signals are received by dual-polarized antennas individually [10]. The difference of SNR is observed to detect the presence of NLOS signal. However, LOS signals may also be attenuated by trees and body masking in certain directions. And the method cannot operate in real time. Furthermore digital 3D 


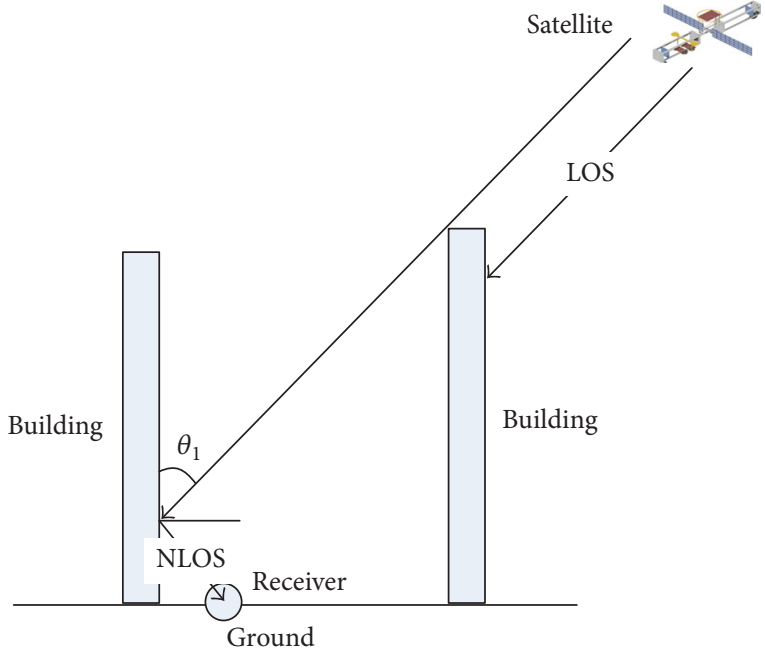

(a)

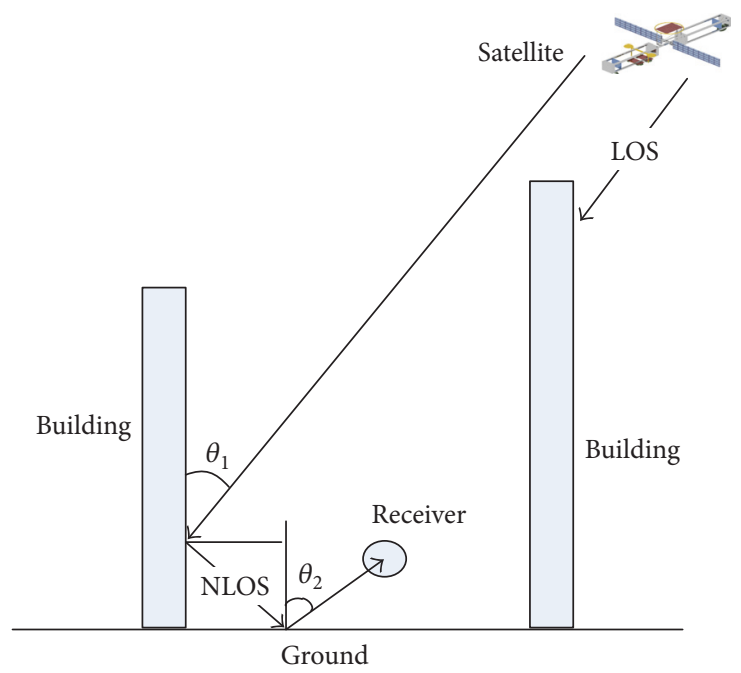

(b)

FIGURE 1: The basic geometry of LOS signal and NLOS signal: single reflection (a) and double reflection (b).

urban models are used to detect and mitigate NLOS signal [11, 12]. These algorithms brought in an increased computational burden. And the urban models for cities are a big task and may be unavailable for common users.

In this paper, the NLOS signal detector based on single orthogonal dual-polarized antenna is proposed without any previous knowledge of its characteristics and an increased computational burden. The antenna is assumed to have two pure linear polarized channels. The patterns of the two channels are assumed to be the same both in gain and in phase and to be azimuth-independent. The polarized diversity between the horizontal polarized and vertical polarized component of RHCP signal is used to remove the LOS signal by parallel cross-cancellation. The NLOS signal can be detected on conventional high-sensitivity receiver in real time.

\section{Refection Characteristics of NLOS Signal}

In the urban canyon and valley, the LOS signal of some satellites is blocked by buildings and other obstacles. Signals from these satellites can only be received via reflections as shown in Figure 1. To estimate the characteristics of reflection, the specular reflection model is widely used. The reflected signal is attenuated after reflection, and the more times the reflections, the weaker the reflected signal. Therefore single reflected signal and double reflected signal are discussed only in this paper.

For any polarized wave, it can be resolved into two orthogonal components, the vertical polarized and horizontal polarized component. The two orthogonal components of incoming wave $E_{i}$ can be expressed as

$$
E_{i}=\left[\begin{array}{c}
E_{i \mathrm{HP}} \\
E_{i \mathrm{VP}}
\end{array}\right]=\left|E_{i}\right|\left[\begin{array}{c}
\cos \gamma \\
\sin \gamma e^{j \eta}
\end{array}\right]
$$

where $E_{i \mathrm{HP}}, E_{i \mathrm{VP}}$ denote the horizontal polarized and vertical polarized component of incoming wave, respectively. Additionally, $\gamma$ denotes the angle between the electric field $E_{i}$ and horizontal polarized component $E_{i \mathrm{HP}}$, while $\eta$ denotes the advance phase of $E_{i \mathrm{VP}}$ versus $E_{i \mathrm{HP}}$. Then $(\gamma, \eta)$ represents the polarization of incoming wave, $\gamma=45^{\circ}$, and $\eta=-90^{\circ}$ for RHCP signal.

The single reflected wave $E_{s}$ and double reflected wave $E_{d}$ can be expressed as

$$
\begin{aligned}
E_{s} & =\left[\begin{array}{c}
E_{s \mathrm{HP}} \\
E_{s \mathrm{VP}}
\end{array}\right]=\left|E_{i}\right| e^{j 2 \pi f \Delta \tau_{s}}\left[\begin{array}{c}
\Gamma_{\mathrm{HP} 1}\left(\theta_{1}\right) \cos \gamma \\
\Gamma_{\mathrm{VP} 1}\left(\theta_{1}\right) \sin \gamma e^{j \eta}
\end{array}\right] \\
E_{d} & =\left[\begin{array}{c}
E_{d \mathrm{HP}} \\
E_{d \mathrm{VP}}
\end{array}\right] \\
& =\left|E_{i}\right| e^{j 2 \pi f \Delta \tau_{d}}\left[\begin{array}{c}
\Gamma_{\mathrm{HP} 1}\left(\theta_{1}\right) \Gamma_{\mathrm{HP} 2}\left(\frac{\pi}{2}-\theta_{2}\right) \cos \gamma \\
\Gamma_{\mathrm{VP} 1}\left(\theta_{1}\right) \Gamma_{\mathrm{VP} 2}\left(\frac{\pi}{2}-\theta_{2}\right) \sin \gamma e^{j \eta}
\end{array}\right] .
\end{aligned}
$$

In (2), $E_{s \mathrm{HP}}, E_{s \mathrm{VP}}, E_{d \mathrm{HP}}$, and $E_{d \mathrm{VP}}$ denote the horizontal polarized and vertical polarized component of single reflected wave and double reflected wave, respectively, where $\Delta \tau_{s}$ and $\Delta \tau_{d}$ denote the extra delay of reflection compared with LOS signal. We have to note that the extra transmission LOS signals is ignored since the additional path is much smaller compared with the distance between satellite and the receiver. For the sake of simplicity, we assume that $\theta_{1}=\theta_{2}$.

The polarization of reflection changes along with the incoming elevation angle, especially at around the Brewster angle. Fresnel formulas can quantify this transformation [13]. 

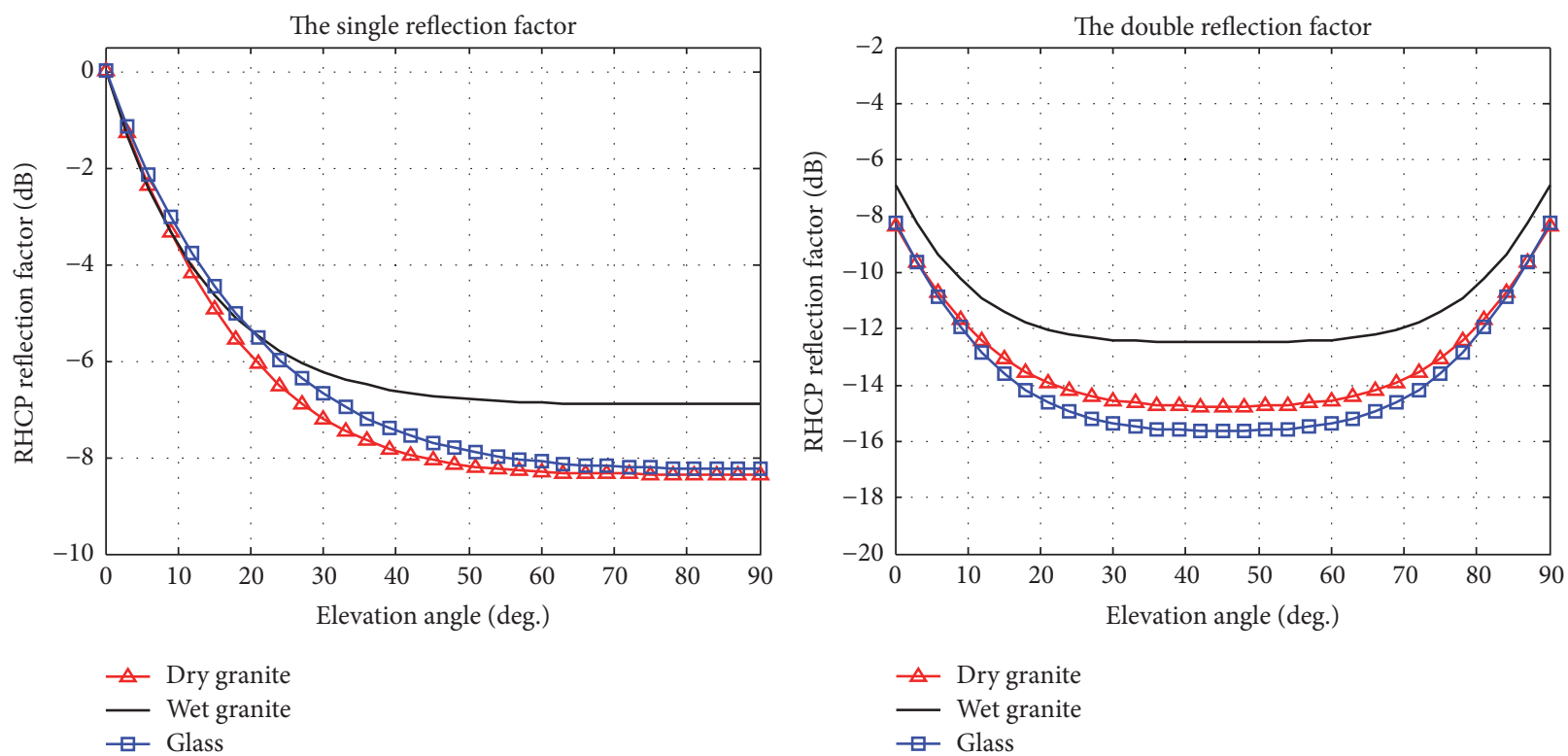

FIGURE 2: The reflection factor of NLOS signal when the LOS signal is RHCP.

Each reflection factor for vertical polarization $\Gamma_{\mathrm{VP} i}$, as well as horizontal polarization $\Gamma_{\mathrm{HP}}$, is given by

$$
\begin{array}{r}
\Gamma_{\mathrm{VP} i}(\theta) \\
=\frac{\left(\varepsilon_{r i}-j \sigma_{i} / f \varepsilon_{0}\right) \sin \theta-\sqrt{\left(\varepsilon_{r i}-j \sigma_{i} / f \varepsilon_{0}\right)-\cos ^{2} \theta}}{\left(\varepsilon_{r}-j \sigma_{i} / f \varepsilon_{0}\right) \sin \theta+\sqrt{\left(\varepsilon_{r i}-j \sigma_{i} / f \varepsilon_{0}\right)-\cos ^{2} \theta}} \\
\Gamma_{\mathrm{HP} i}(\theta)=\frac{\sin \theta-\sqrt{\left(\varepsilon_{r i}-j \sigma_{i} / f \varepsilon_{0}\right)-\cos ^{2} \theta}}{\sin \theta+\sqrt{\left(\varepsilon_{r i}-j \sigma_{i} / f \varepsilon_{0}\right)-\cos ^{2} \theta}},
\end{array}
$$

where $\varepsilon_{0}$ denotes free space dielectric constant, $\varepsilon_{r i}$ denotes relative dielectric constant, $\sigma_{i}$ is conductivity of reflector, $f$ is radian frequency, and $\theta$ represents the incoming elevation angle.

In the urban canyon, granite and glass are common reflector, so the granite and glass are used to calculate the reflection factor. Assume that LOS signal is ideal RHCP. The RHCP reflection factors are shown in Figure 2.

In Figure 2, the magnitude of reflection factor for RHCP signal reduces when the incoming elevation angle increases in the case of single reflection. The reflection phase for the vertically polarized component varies $180^{\circ}$ across the Brewster angle. For dry granite, the Brewster angle is about $24^{\circ}$ while it is $21^{\circ}$ and $25^{\circ}$ for wet granite and glass. At angles near the horizon RHCP is reflected as RHCP. At the Brewster angle the reflected signal is essentially horizontally polarized. At angles near zenith, RHCP is reflected as LHCP. In the situation of double reflection, it is complex since there are two reflectors. The polarization of incoming wave changes frequently. But we have to note that the reflection factor is under $-12 \mathrm{~dB}$ when the incoming elevation angle is between $20^{\circ}$ and $70^{\circ}$.
Take the ratio of vertical polarized and horizontal polarized component to denote the axial ratio of the reflection; the results are shown in Figure 3. The magnitude of the vertical polarized and horizontal polarized component for LOS signal is nearly equal. But the balance is broken when signal is reflected. Around the Brewster angle the axial ratio is worse seriously.

In the case of single reflection, the axial ratio changes slowly while the reflection factor is much smaller at high elevation angle. At low elevation angle, the reflection factor of the NLOS signal is nearly equivalent to that of the LOS signal, but the axial ratio changes acutely. In the case of double reflection, the axial ratio and reflection factor are symmetrical. And the reflection factor at the elevation angle $\left[20^{\circ}, 70^{\circ}\right]$ is much small. Take both Figures 2 and 3 into consideration; the analysis can be summarized as follows:

(i) As it known, at elevation angle lower than $10^{\circ}$ the NLOS signal can hardly appear and signal coming from the elevation angle higher than $80^{\circ}$ may be abandoned because of poor quality. So single reflection from $\left[10^{\circ}, 80^{\circ}\right]$ and double reflection from $\left[10^{\circ}, 20^{\circ}\right]$ and $\left[70^{\circ}, 80^{\circ}\right]$ are analyzed.

(ii) Since the strong NLOS signal at around the Brewster angle is our target to detect, the worsened axial ratio can be used to distinguish the NLOS signal from LOS signal when the vertical polarized and horizontal polarized component can be received individually.

\section{NLOS Signal Detection Based on Orthogonal Dual-Polarized Antenna}

NLOS signal detection can be achieved when the vertical polarized and horizontal polarized components of RHCP are received individually. Parallel cross-cancellation receiver as 

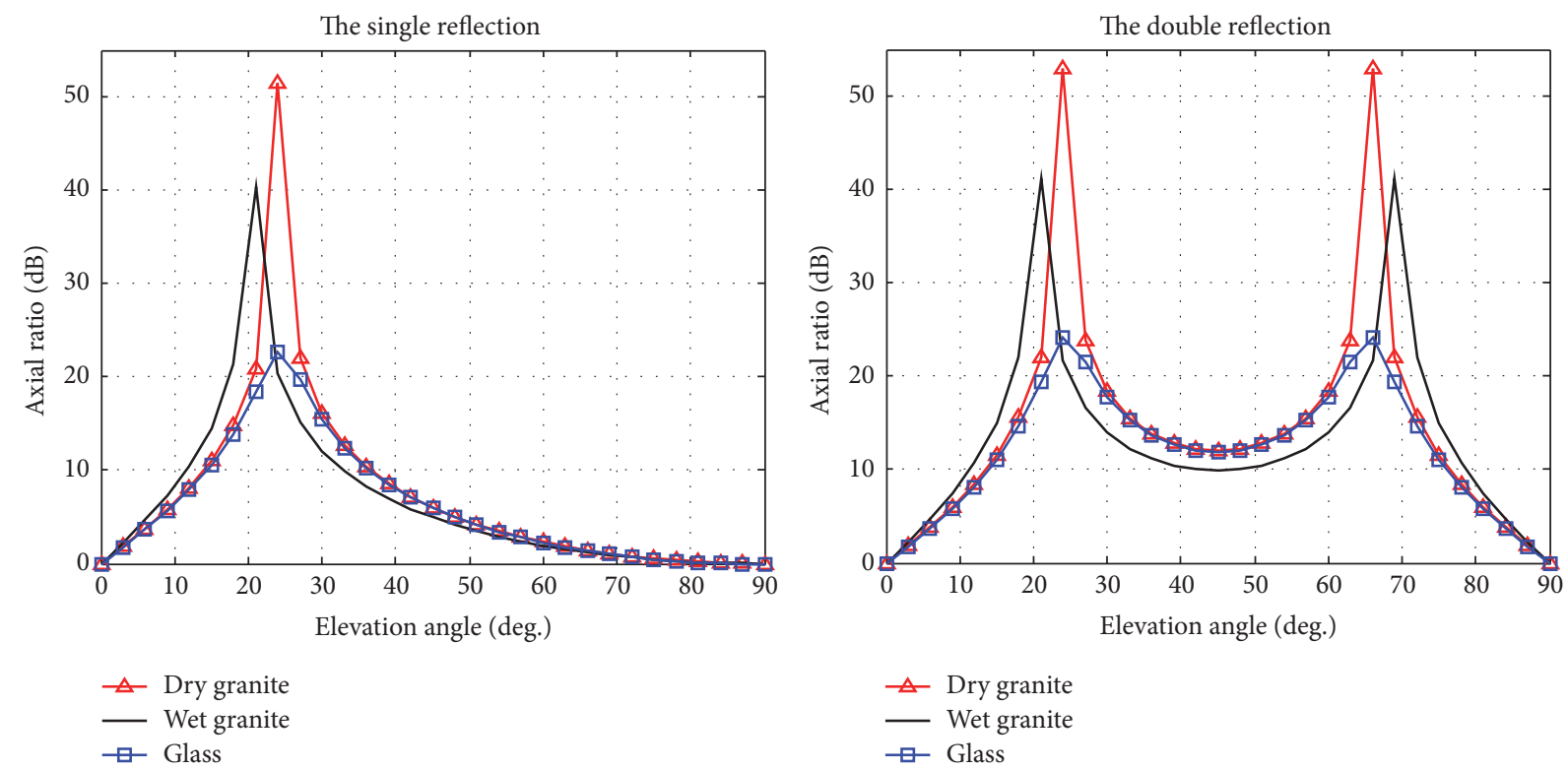

FIGURE 3: The axial ratio of NLOS signal when the LOS signal is RHCP.

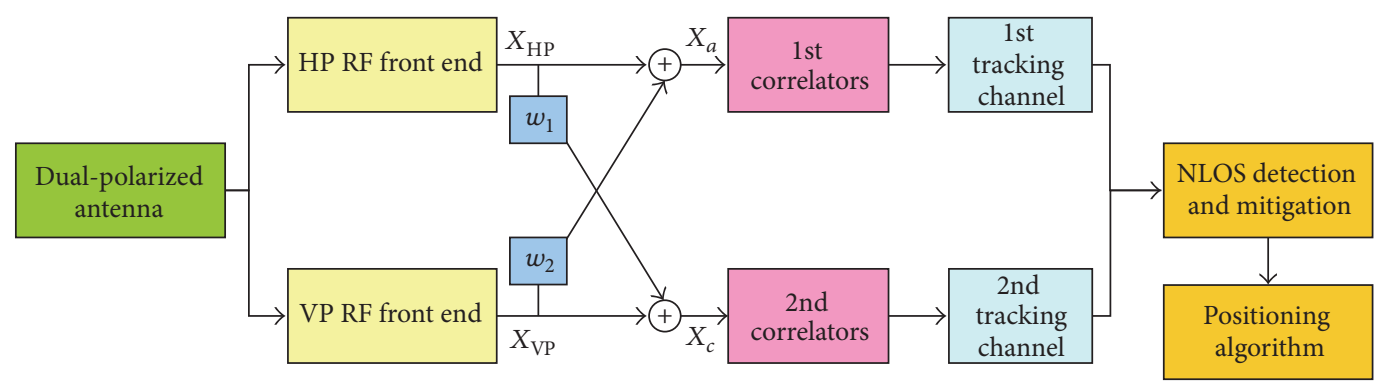

FIGURE 4: Parallel cross-cancellation receiver with orthogonal dual-polarized antenna.

well as its signal model is introduced in this section. Parallel cross-cancellation is used to weaken LOS signal in one channel so that only NLOS signal remains. Results of the two parallel channels would determine the presence of NLOS signal.

The following assumptions are made to simplify our analysis, and all the following analysis is based on the special case meeting the assumptions.

(i) The additional delay brought in by NLOS signal is long delay and is larger than one code chip period. It is reasonable that the long delay can significantly degrade the positioning accuracy.

(ii) If the NLOS signal is composed of both single reflection and double reflection, the path delay between the two signals should be less than one code chip period. It is a fair assumption that one of the two reflections may be mitigated by multipath mitigation technique if the path delay is too long, such as narrow correlation.

(iii) The antenna has two pure linear polarized channels to receive the vertical polarized and horizontal polarized component of incoming signal, respectively. The patterns of the two channels are the same as both in gain and in phase and are azimuth-independent.

3.1. Signal Model. The orthogonal dual-polarized antenna has two ports to receive the vertical polarized (VP) and horizontal polarized (HP) signal individually. In the parallel cross-cancellation receiver as shown in Figure 4, each port is followed by an independent RF front end. Parallel cross-cancellation can be achieved by the two parallel receiving channels. The residual signals are then transmitted to correlators and tracking channel. NLOS signal detection and mitigation before positioning algorithm are based on the results of the two parallel channels.

The LOS signal $x_{i}(t)$ and NLOS signal $x_{m}(t)$ can be expressed as

$$
\begin{gathered}
x_{i}(t)=s(t) \cdot\left(\cos \gamma+\sin \gamma e^{j \eta}\right)=x_{i \mathrm{HP}}(t)+x_{i \mathrm{VP}}(t) \\
x_{m}(t)=x_{s}(t)+x_{d}(t)=s(t)\left[e^{-j 2 \pi f \Delta \tau_{1}}\right. \\
\cdot\left(\cos \gamma \cdot \Gamma_{1 \mathrm{HP}}+\sin \gamma e^{j \eta} \cdot \Gamma_{1 \mathrm{VP}}\right)+e^{-j 2 \pi f \Delta \tau_{2}}
\end{gathered}
$$




$$
\begin{aligned}
& \left.\cdot\left(\cos \gamma \cdot \Gamma_{1 \mathrm{HP}} \cdot \Gamma_{2 \mathrm{HP}}+\sin \gamma e^{j \eta} \cdot \Gamma_{1 \mathrm{VP}} \cdot \Gamma_{2 \mathrm{VP}}\right)\right] \\
& =x_{s \mathrm{HP}}(t)+x_{s \mathrm{VP}}(t)+x_{d \mathrm{HP}}(t)+x_{d \mathrm{VP}}(t),
\end{aligned}
$$

where $x_{i \mathrm{HP}}(t), x_{s \mathrm{VP}}(t), x_{m \mathrm{HP}}(t)$, and $x_{m \mathrm{VP}}(t)$ denote the vertical polarized and horizontal polarized component of LOS signal and NLOS signal, respectively. And $x_{s}(t), x_{d}(t)$ denote the single reflection and double reflection. $s(t)$ represents the LOS signal arriving at the receiver and we have

$$
s(t)=A p\left(t-\tau_{0}\right) \cos \left(2 \pi f t+\phi_{0}\right)
$$

where $A$ denotes the magnitude, $p(t)$ denotes the pseudorandom code, $\tau_{0}$ denotes the propagation delay of LOS signal, and $\phi_{0}$ denotes the carrier phase of LOS signal.

For the sake of simplicity, only one satellite signal is considered. Then the signal received by vertical polarized port $X_{\mathrm{VP}}$ and horizontal polarized port $X_{\mathrm{HP}}$ can be expressed as

$$
\begin{aligned}
& X_{\mathrm{HP}}=k G\left(\theta_{1}\right) x_{i \mathrm{HP}}+G\left(\theta_{1}\right) x_{s \mathrm{HP}}+G\left(\pi-\theta_{1}\right) x_{d \mathrm{HP}} \\
& +n_{1}=s(t) \cos \gamma\left[k G\left(\theta_{1}\right)+G\left(\theta_{1}\right) \Gamma_{1 \mathrm{HP}} e^{-j 2 \pi f \Delta \tau_{1}}\right. \\
& \left.+G\left(\pi-\theta_{1}\right) \Gamma_{1 \mathrm{HP}} \Gamma_{2 \mathrm{HP}} e^{-j 2 \pi f \Delta \tau_{2}}\right]+n_{1} \\
& X_{\mathrm{VP}}=k G\left(\theta_{1}\right) x_{i \mathrm{VP}}+G\left(\theta_{1}\right) x_{s \mathrm{VP}}+G\left(\pi-\theta_{1}\right) x_{d \mathrm{VP}} \\
& +n_{2}=s(t) \sin \gamma e^{j \eta}\left[k G\left(\theta_{1}\right)+G\left(\theta_{1}\right) \Gamma_{1 \mathrm{HP}} e^{-j 2 \pi f \Delta \tau_{1}}\right. \\
& \left.+G\left(\pi-\theta_{1}\right) \Gamma_{1 \mathrm{HP}} \Gamma_{2 \mathrm{HP}} e^{-j 2 \pi f \Delta \tau_{2}}\right]+n_{2},
\end{aligned}
$$

when $k=0$, the reflection acts as NLOS signal, else it acts as multipath. $G\left(\theta_{1}\right)$ and $G\left(\pi-\theta_{1}\right)$ denote the gain of antenna at the incoming elevation angle $\theta_{1}$ and $\pi-\theta_{1} . n_{1}$ and $n_{2}$ denote the white Gaussian noise with zero mean.

As shown in Figure 4, the signal after parallel crosscancellation is

$$
\begin{aligned}
X_{c}(t)= & w_{1} X_{\mathrm{HP}}+X_{\mathrm{VP}} \\
= & k G\left(\theta_{1}\right) s(t) e_{c i}+G\left(\theta_{1}\right) s(t) \cdot e^{-j 2 \pi f \Delta \tau_{1}} e_{c s} \\
& +G\left(\pi-\theta_{1}\right) s(t) \cdot e^{-j 2 \pi f \Delta \tau_{2}} e_{c d}+n_{3} \\
X_{a}(t)= & X_{\mathrm{HP}}+w_{2} X_{\mathrm{VP}} \\
= & k G\left(\theta_{1}\right) s(t) e_{a i}+G\left(\theta_{1}\right) s(t) \cdot e^{-j 2 \pi f \Delta \tau_{1}} e_{a s} \\
& +G\left(\pi-\theta_{1}\right) s(t) \cdot e^{-j 2 \pi f \Delta \tau_{2}} e_{a d}+n_{4} .
\end{aligned}
$$

In the right of (7) and (8), the first term is the residual signal of LOS signal, while the second and the third terms are the residual signal of NLOS signal. And $e_{c i}, e_{c s}, e_{c d}, e_{a i}, e_{a s}$, and $e_{a d}$ represent the complex residual factor of LOS signal, single reflected signal, and double reflected signal, respectively.
The ideal RHCP signal can be expressed with $\gamma=45^{\circ}$, $\eta=-90^{\circ}$. However, the actual GNSS signal is not an ideal RHCP signal, so the polarization has some bias. The bias of polarization is defined as

$$
\begin{aligned}
& \gamma=\frac{\pi}{4}+\Delta \gamma \\
& \eta=\frac{\pi}{2}+\Delta \eta
\end{aligned}
$$

where $(\Delta \gamma, \Delta \eta)$ represents the nonideal characteristic of actual GNSS signal.

3.2. NLOS Signal Detection. NLOS signal detection firstly has to remove the LOS signal from the received signal. The proposed method is to adjust the weight to make sure the power of residual LOS signal is small enough into the 2nd correlator, while the power of residual signal into the 1st correlator must be equal to conventional GNSS receiver. It is accomplished by

$$
\begin{gathered}
\min _{w_{1}}\left\{e_{c i}\right\} \\
e_{a i}\left(w_{2}\right) \propto \sqrt{2} .
\end{gathered}
$$

Usually there is no prior knowledge about the polarization of the LOS signal, so the weights are simply adjusted as

$$
w_{1}=w_{2}=e^{-j \pi / 2} .
$$

Then

$$
\begin{aligned}
& e_{c i}=\sqrt{1-\cos (2 \Delta \gamma) \cos \Delta \eta} \\
& e_{a i}=\cos \gamma+\sin \gamma e^{j \Delta \eta} .
\end{aligned}
$$

Since the transmitted GNSS signal is RHCP, $(\Delta \gamma, \Delta \eta)$ is much small. According to the residual factor in (12), the LOS signal is weakened in the 2nd correlator while it is preserved in the 1st correlator. So the orthogonal dual-polarized antenna acts as a conventional RHCP antenna in the 1st correlator. The correlation in the 1 st and 2 nd correlator can be expressed as

$$
\begin{aligned}
& I R_{c p}(\varepsilon) \\
& =k G\left(\theta_{1}\right) A T_{c} R_{e s}(\varepsilon-\Delta \tau) e_{c i} \cos \left(\phi_{e}\right) \\
& \quad+G\left(\theta_{1}\right) A T_{c} R_{e s}\left(\varepsilon-\Delta \tau_{1}\right) e_{c s} \cos \left(\phi_{e}+\Delta \phi_{1}\right) \\
& \quad+G\left(\pi-\theta_{1}\right) A T_{c} R_{e s}\left(\varepsilon-\Delta \tau_{2}\right) e_{c d} \cos \left(\phi_{e}+\Delta \phi_{2}\right) \\
& I R_{a p}(\varepsilon) \\
& =k G\left(\theta_{1}\right) A T_{c} R_{e s}(\varepsilon-\Delta \tau) e_{a i} \cos \left(\phi_{e}\right) \\
& \quad+G\left(\theta_{1}\right) A T_{c} R_{e s}\left(\varepsilon-\Delta \tau_{1}\right) e_{a s} \cos \left(\phi_{e}+\Delta \phi_{1}\right) \\
& \quad+G\left(\pi-\theta_{1}\right) A T_{c} R_{e s}\left(\varepsilon-\Delta \tau_{2}\right) e_{a d} \cos \left(\phi_{e}+\Delta \phi_{2}\right),
\end{aligned}
$$

where $T_{c}$ represents chip period, $\phi_{e}$ denotes the residual carrier phase, and $\Delta \phi_{1} \Delta \phi_{2}$ denotes the extra phase by reflection. 


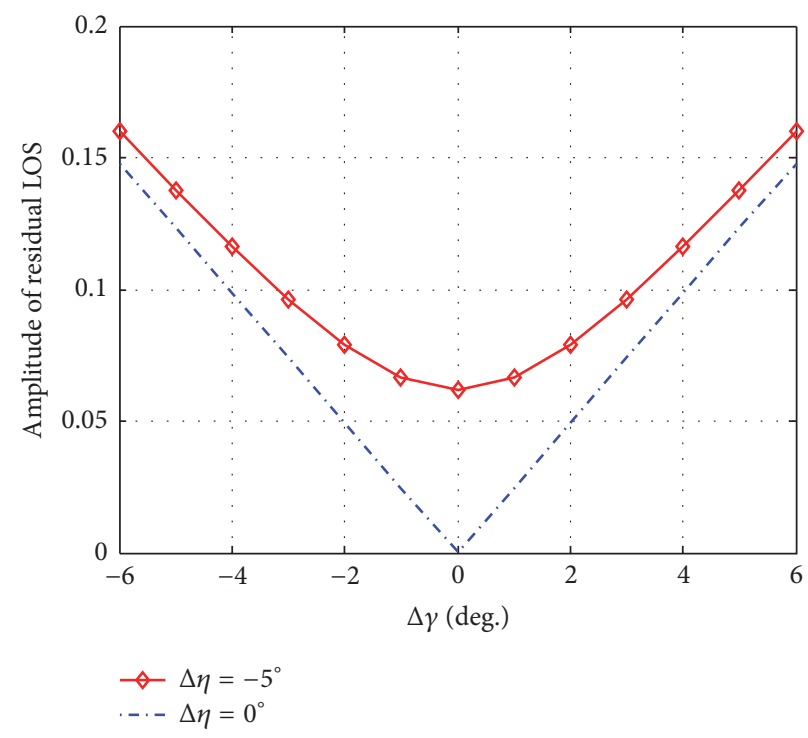

FIgURE 5: The magnitude of residual LOS signal.

$R_{e s}(\varepsilon)$ denotes the correlation of the pseudorandom code. In the right of (13) and (14), the first term is the correlation of LOS signal, while the second and the third terms are correlation of NLOS signal. Because of cross-cancellation, the first term in the right of (13) tends to be much small. When the threshold of code acquisition is set to be reasonable, LOS signal cannot be acquired in the 2 nd correlator even if it is present.

Above all, once the LOS signal is present, the 1st channel is locked on LOS signal, but the 2nd channel can only lock on NLOS signal or multipath. So there are three kinds of signal that can be detected:

(i) LOS signal only: satellite signal can only be acquired in the 1st correlator.

(ii) LOS signal and multipath with long delay: satellite signal can be acquired in both of correlators, but the difference of pseudoranges between the two channel is large.

(iii) NLOS signal: satellite signal can be acquired in both of correlators, but the pseudoranges of the two channels are nearly equal.

\section{Simulation and Results}

To verify the effectiveness of the proposed method, simulation is carried out based on GPS signals. According to interface control document of GPS (IS-GPS-200H 2013), for the angular range of \pm 13.8 degrees from nadir, the axial ratio of $\mathrm{L} 1$ shall be no worse than $1.2 \mathrm{~dB}$ for Block IIA and shall be no worse than $1.8 \mathrm{~dB}$ for Block IIR/IIR-M/IIF/GPS III SVs. So the range of $\Delta \gamma$ is set to be $|\Delta \gamma| \leq 6^{\circ}$ and $|\Delta \eta| \leq 5^{\circ}$. Firstly, the magnitude of residual signal is calculated. Then the correlation curve and phase-discrimination curve using the regular DLL are simulated at different incoming elevation.
4.1. The Numerical Analysis of Residual Signal. NLOS signal detection is mainly based on that the magnitude of residual NLOS signal is much larger than that of residual LOS signal after parallel cross-cancellation in 2nd correlator. This is the key point of the proposed method. So the magnitude of residual signal is simulated to verify the effectiveness of the proposed method.

The numerical analysis was carried out adequately and all results are normalized. The magnitude of residual LOS signal is shown in Figure 5.

In Figure 5, the magnitude of residual LOS signal is only the function of polarization of signal. The magnitude of residual LOS signal is always smaller than 0.16 . It is influenced by the bias of $\gamma$ seriously but without the influence of incoming elevation angle. The magnitude of residual LOS signals is small when the axial ratio of LOS signals is small. However the residual NLOS signal is also affected by the incoming elevation angle. The worst results when $\Delta \gamma=-6^{\circ}$ are shown in Figure 6.

In Figure 6, because of single reflection, the magnitude of residual NLOS signal varies while the elevation angle is changing. The magnitude is larger when in a higher elevation angle. The reflection phase for the vertically polarized component varies $180^{\circ}$ across the Brewster angle. So the two orthogonal polarized components tend to be out of phase after weighting. The processing of cancellation results in the strengthening of residual NLOS signal in fact. But the strong residual NLOS signal only appears at the elevation angle $\left[10^{\circ}\right.$, $\left.20^{\circ}\right]$ and $\left[70^{\circ}, 80^{\circ}\right]$ after double reflection.

Obviously the magnitude of residual NLOS signal is larger than 0.2 while the residual LOS signal is smaller than 0.16 . Moreover if the axial ratio of satellite signal is smaller than $1.2 \mathrm{~dB}$, the magnitude difference between residual NLOS signal and LOS signal is larger.

When the incoming elevation angle is the same, the residual multipath is weaker in glass than the other two reflectors. 

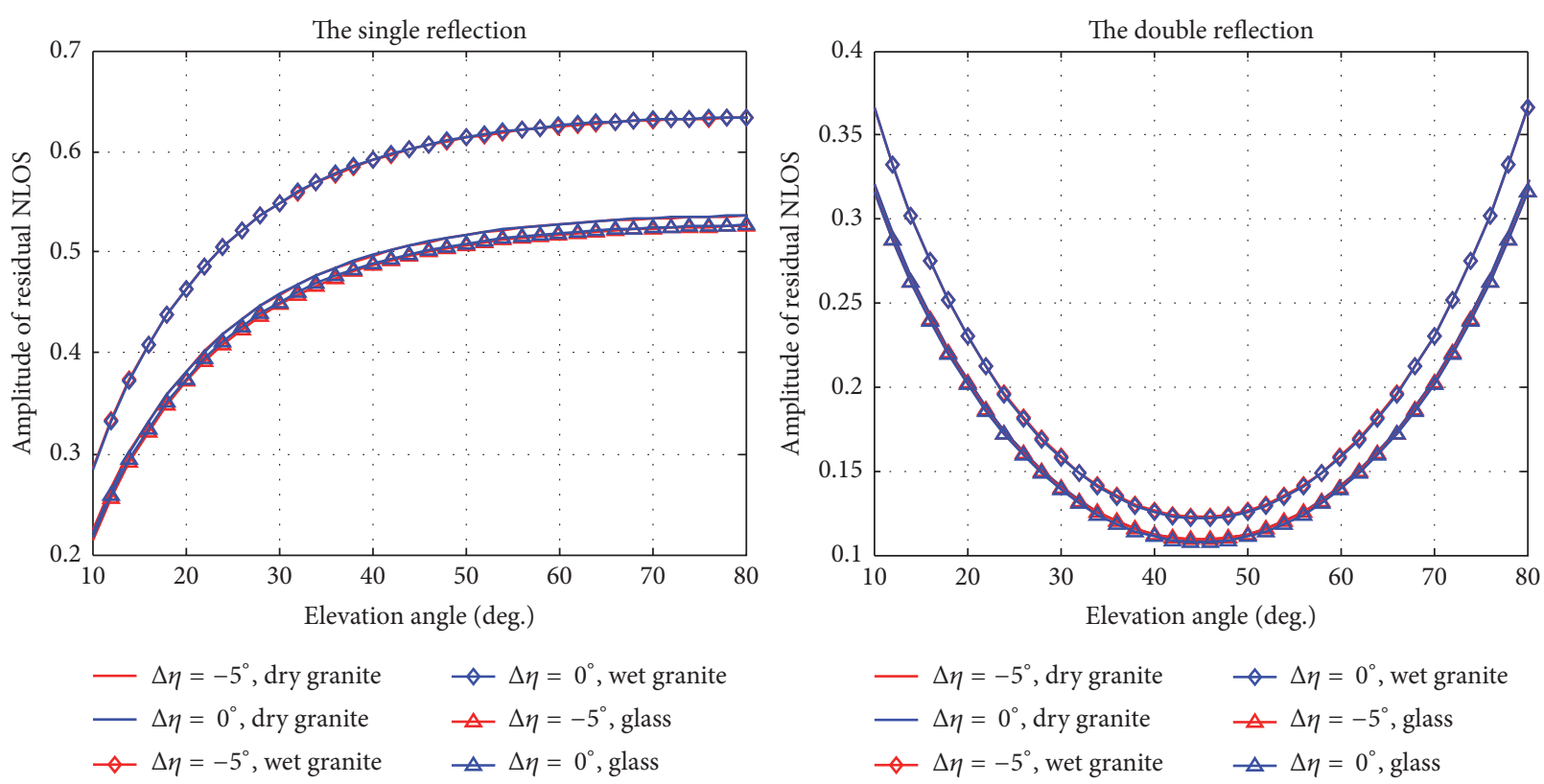

FIgURE 6: The magnitude of residual NLOS signal.

So the performance of multipath detection is only analyzed in the condition of glass in the following section.

4.2. The Performance for NLOS Signal Detection. The code tracking loop in the GPS receiver is a delay lock loop (DLL) called an early-late tracking loop [14]. The performance for NLOS signal detection is shown by the correlation results in DLL.

In the first scenario, the incoming elevation angle of LOS signal is $10^{\circ}$ and the extra delay of single reflection is set to be $1.2 T_{c}$ while the extra delay of double reflection is set to be $1.5 T_{c}$. The reflected factor is calculated when $\Delta \gamma=-6^{\circ}, \Delta \eta=$ $-5^{\circ}$. If the LOS signal is present, the single and double reflections acted as multipath, or else they are NLOS signal. The RF front end is treated to be ideal and the error of carrier tracking loop is ignored; the correlation curve and phase-discrimination curve are shown in Figure 7.

In Figure 7, the correlation curve and phase-discrimination curve of conventional GNSS receiver are shown in 1st correlator and 1st DLL, and receiver locked on the LOS signal with multipath if LOS signal presents, or else it locked on NLOS signal. Since the coordinate on the $\mathrm{x}$-axes where the phase-discrimination curve crosses the zero point of the $y$ axes represents the error of measured pseudorange, the error caused by multipath is shown.

But in 2nd correlator and 2nd DLL the residual LOS signal after cross-cancellation is weakened heavily. And the curve of residual signal is mainly dependent on the characteristic of multipath or NLOS signal. Once the threshold of code acquisition is set to be larger than residual LOS signal but smaller than single reflected NLOS signal, the 2nd correlator cannot acquire any LOS signal.

If the receiving signal is composed of NLOS signal only, the 1st correlator can acquire the satellite signal but the 2 nd correlator cannot. If the receiving signal is composed of LOS signal with long delay multipath, the two correlators can acquire the satellite signal but pseudoranges difference between the two signals is larger than one code chip period. If the receiving signal is composed of mixed NLOS signal, the two correlators can acquire the satellite signal but pseudoranges difference between the two signals is less than one code chip period. And if NLOS signal is only one kind of reflection, the pseudoranges of the two signals are nearly equal. So the NLOS signal is detected.

Then the incoming elevation angle of LOS signal is set to be $20^{\circ}$, the correlation curve and phase-discrimination curve are shown in Figure 8. Obviously, the difference between Figures 7 and 8 is the correlation results of single reflected NLOS signal and double reflected NLOS signal. The 2 nd correlator also cannot acquire any LOS signal. So the NLOS signal is detected if it is present.

The simulation results and their analysis can be summarized as follows:

(i) When the incoming elevation angle is higher than $10^{\circ}$, the residual single reflected NLOS signal is stronger than the residual LOS signal. And the higher the elevation angle, the stronger the residual single reflected NLOS signal. The strong residual double reflected NLOS signal only appears at the elevation angles $\left[10^{\circ}\right.$, $\left.20^{\circ}\right]$ and $\left[70^{\circ}, 80^{\circ}\right]$.

(ii) With parallel cross-cancellation, LOS signal in the 2nd correlator is too weakened to be acquired while it plays an important part in the 1st correlator once it is present.

(iii) When only the 1st correlator can acquire satellite signal, LOS signal is present. When the two correlators can acquire the satellite signal but pseudoranges 

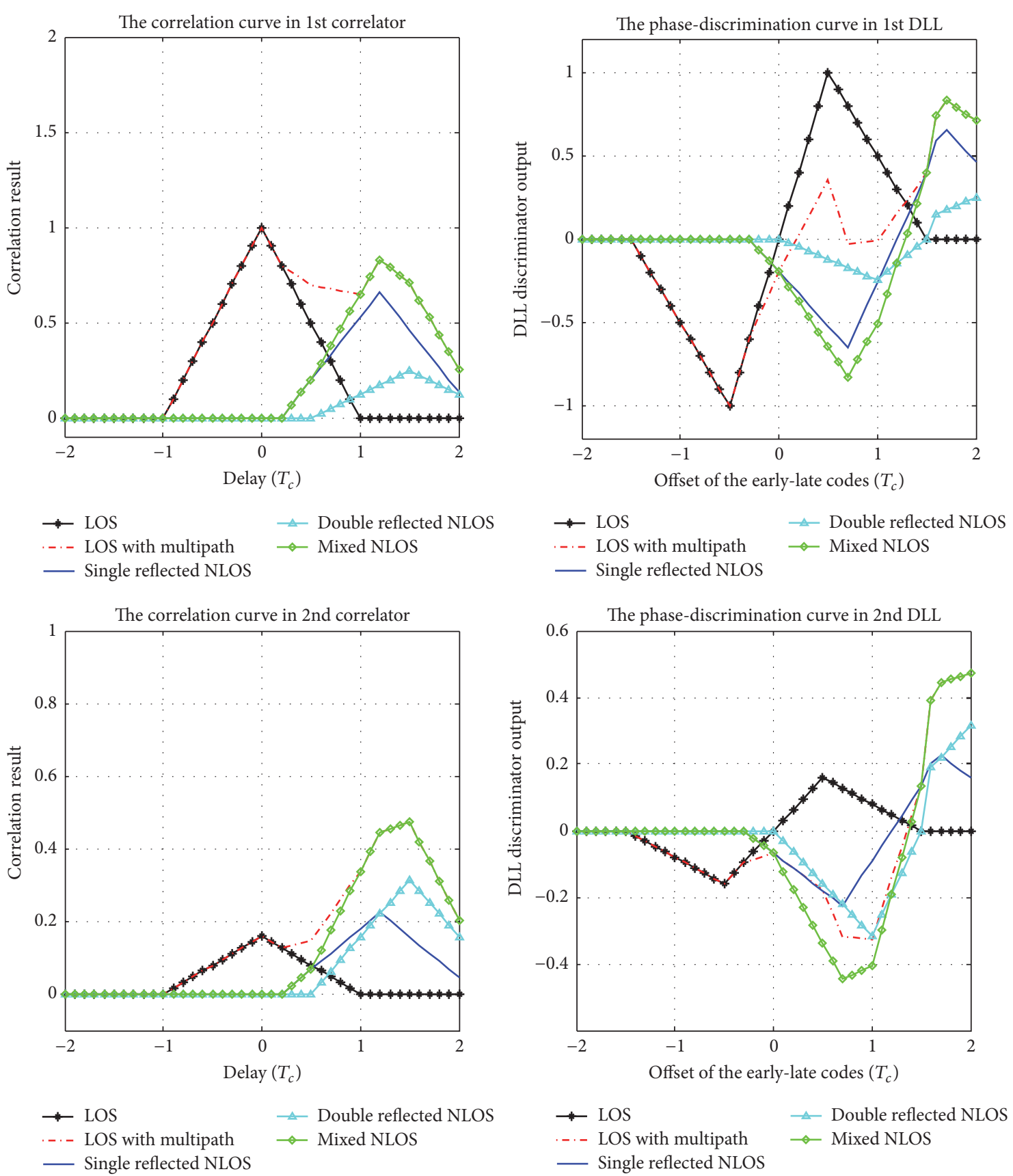

FIGURE 7: Correlation results for parallel cross-cancellation receiver at elevation $10^{\circ}$.

difference between the two signals is larger than one code chip period, LOS signal as well as long delay multipath is present. When the two correlators can acquire the satellite signal but pseudoranges difference between the two signals is less than one code chip period, NLOS signal is present.

\section{Conclusions}

Based on the diversity of the axial ratio between the LOS and NLOS component of RHCP signal, the LOS signal is removed by parallel cross-cancellation. The LOS signal with long delay multipath and NLOS signal can be detected by parallel crosscancellation receiver in real time. The proposed method is based on a theoretical model of orthogonal dual-polarized antenna and without any previous knowledge of incoming signal. The parallel cross-cancellation receiver can be used in urban canyon and valley to decrease the influence of NLOS signal. For future work the actual orthogonal dual-polarized antenna for practical implementation requires further analysis. 

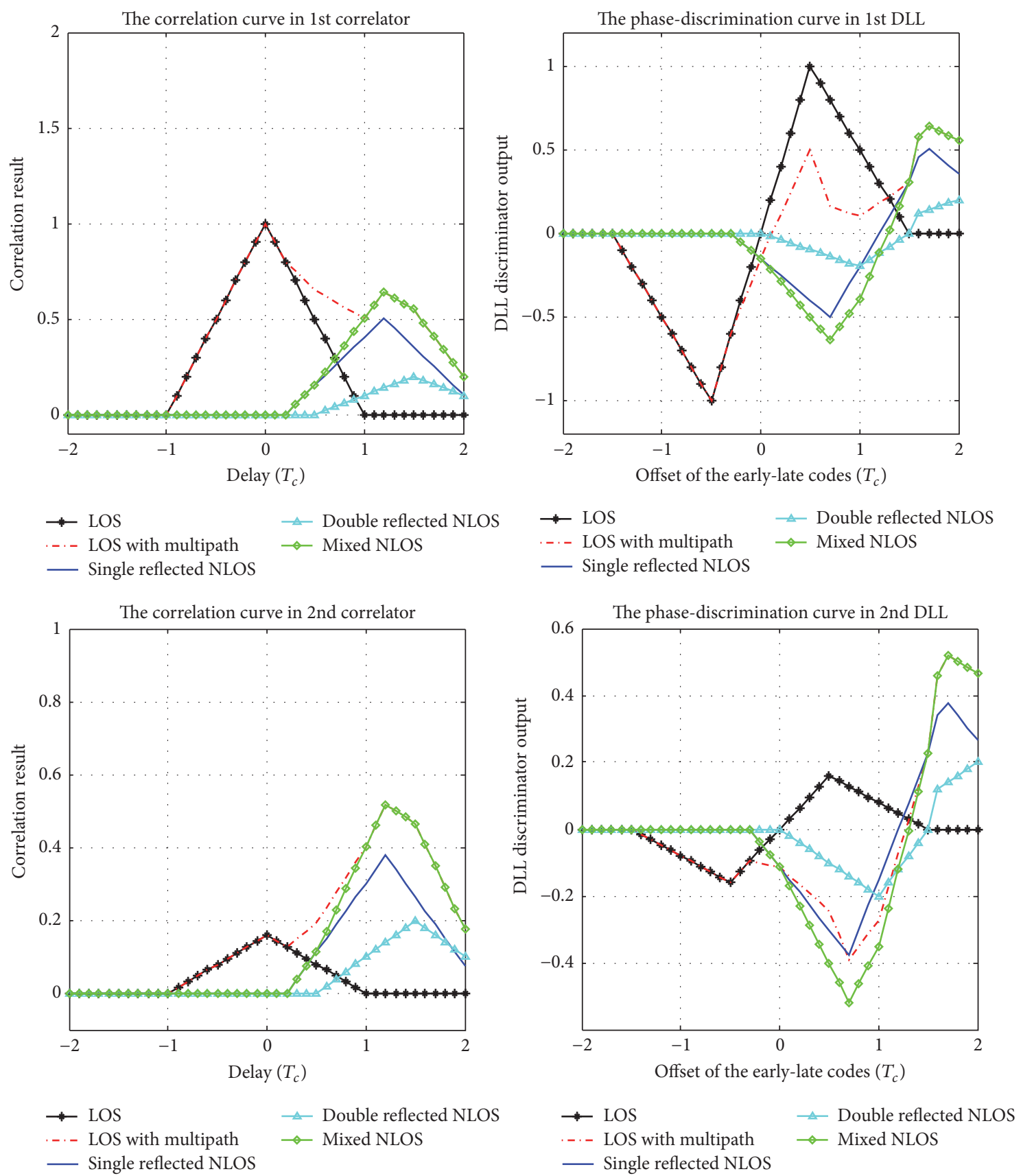

Figure 8: Correlation results for parallel cross-cancellation receiver at elevation $20^{\circ}$.

\section{Conflicts of Interest}

The authors declare that they have no conflicts of interest.

\section{Acknowledgments}

Funding was provided by National Natural Science Foundation of China (Grant no. 61403413).

\section{References}

[1] G. McGraw and M. Braash, "GNSS multipath mitigation using gated and high resolution correlator concepts," in Proceedings of the International Technical Meeting of the Institute of Navigation, pp. 333-342, San Diego, Calif, USA, January 2009.

[2] M. Li, W. Zhao, L. Yuan, and Q. L. Liu, "A GNSS multipath detecting method based on antenna arrays," in Proceedings of the 6th China Satellite Navigation Conference, pp. 319-330, Xi'an, China, May 2015. 
[3] S. Daneshmand, A. Broumandan, N. Sokhandan, and G. Lachapelle, "GNSS multipath mitigation with a moving antenna array," IEEE Transactions on Aerospace and Electronic Systems, vol. 49, no. 1, pp. 693-698, 2013.

[4] J. LaMance and D. Small, "Locata correlator-based beam forming antenna technology for precise indoor positioning and attitude," in Proceedings of the 24th International Technical Meeting of the Satellite Division of the Institute of Navigation (ION GNSS '11), pp. 2436-2445, Portland, Ore, USA, September 2011.

[5] L. Sun, J. P. Chen, S. S. Tan, and Z. Chai, "Research on multipath limiting antenna array with fixed phase center," GPS Solutions, vol. 19, no. 4, pp. 505-510, 2015.

[6] Z. Y. Jiang and P. D. Groves, "GNSS NLOS signal and multipath error mitigation using advanced multi-constellation consistency checking with height aiding," in Proceedings of the 25th International Technical Meeting of the Satellite Division of the Institute of Navigation, pp. 79-88, Nashville, Tenn, USA, September 2012.

[7] C. Cheng, J.-Y. Tourneret, Q. Pan, and V. Calmettes, "Detecting, estimating and correcting multipath biases affecting GNSS signals using a marginalized likelihood ratio-based method," Signal Processing, vol. 118, pp. 221-234, 2016.

[8] L.-T. Hsu, Y. Gu, and S. Kamijo, "NLOS correction/exclusion for GNSS measurement using RAIM and city building models," Sensors, vol. 15, no. 7, pp. 17329-17349, 2015.

[9] M. Brenneman, J. Morton, C. Yang, and F. V. Graas, "Mitigation of GPS multipath using polarization and spatial diversities," in Proceedings of the 20th International Technical Meeting of the Satellite Division of the Institute of Navigation, pp. 1221-1229, Fort Worth, Tex, USA, September 2007.

[10] Z. Y. Jiang and P. D. Groves, "NLOS GPS signal detection using a dual-polarisation antenna," GPS Solutions, vol. 18, no. 1, pp. 15-26, 2014.

[11] D. Betaille, F. Peyret, M. Ortiz, S. Miquel, and L. Fontenay, "A new modeling based on urban trenches to improve GNSS positioning quality of service in cities," IEEE Intelligent Transportation Systems Magazine, vol. 5, no. 3, pp. 59-70, 2013.

[12] P. D. Groves, Z. Jiang, L. Wang, and M. Ziebart, "Intelligent urban positioning using multi-constellation GNSS with 3D mapping and NLOS Signal detection," in Proceedings of the 25th International Technical Meeting of the Satellite Division of the Institute of Navigation (ION GNSS '12), pp. 458-472, Nashville, Tenn, USA, September 2012.

[13] A. Leick, L. Rapoport, and D. Tatarnikov, GPS Satellite Surveying, John Wiley \& Sons, Hoboken, NJ, USA, 4th edition, 2015.

[14] D. K. Elliott and J. H. Christopher, Understanding GPS: Principles and Application, Artech House Inc, Boston, Mass, USA, 2nd edition, 2006. 


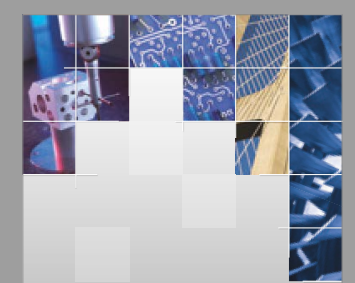

\section{Enfincering}
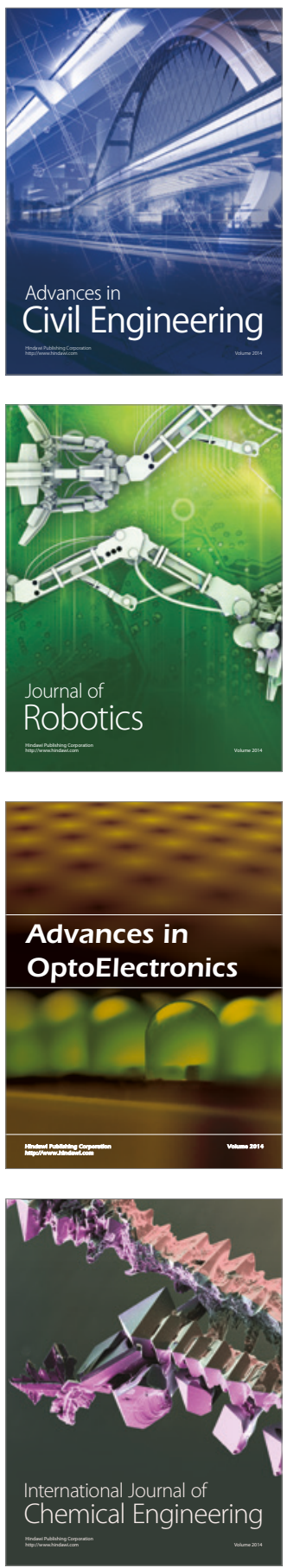

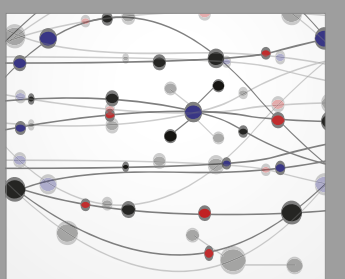

The Scientific World Journal

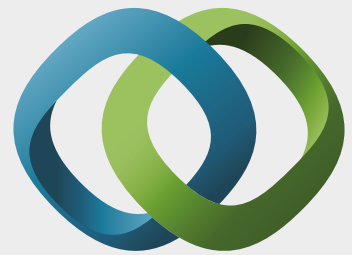

\section{Hindawi}

Submit your manuscripts at

https://www.hindawi.com
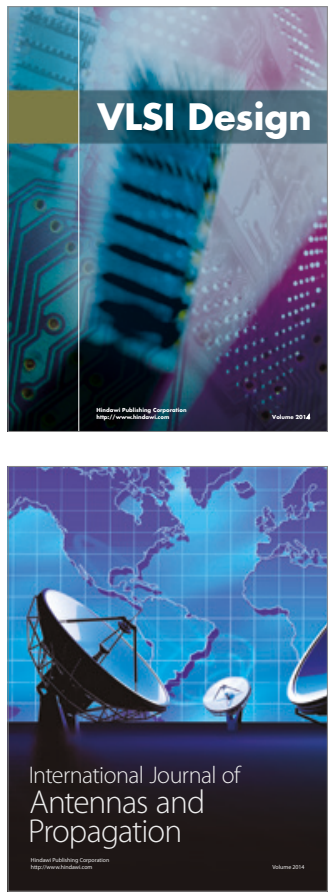

\section{Rotating}

Machinery
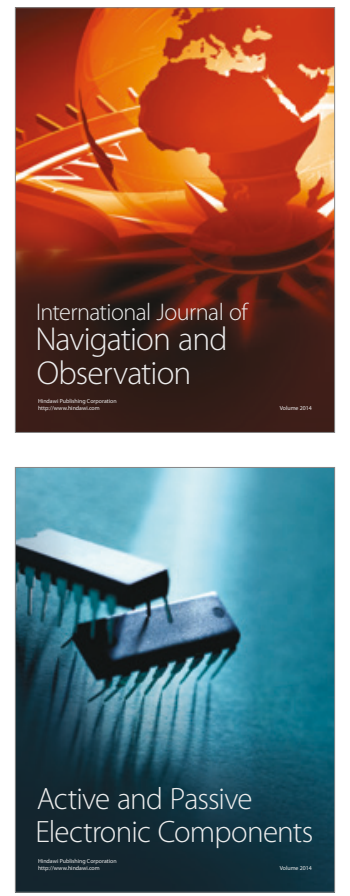
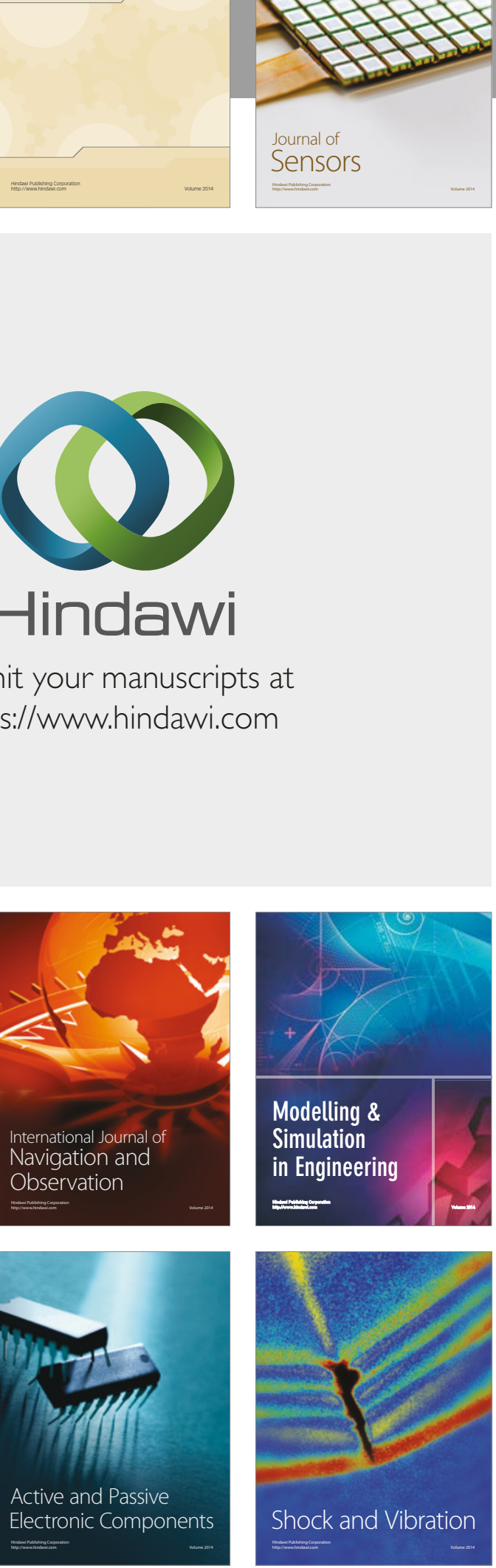
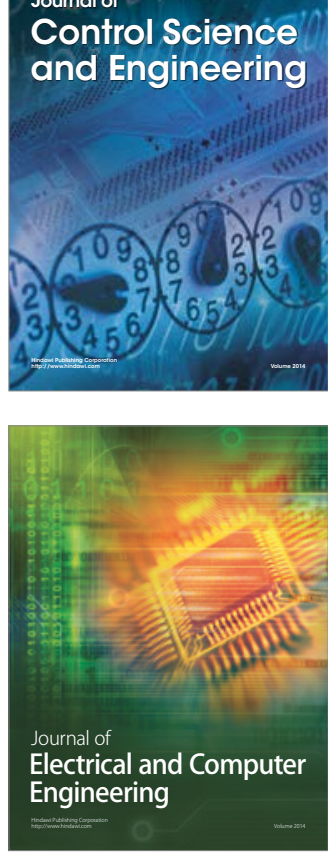

Distributed

Journal of

Control Science

and Engineering
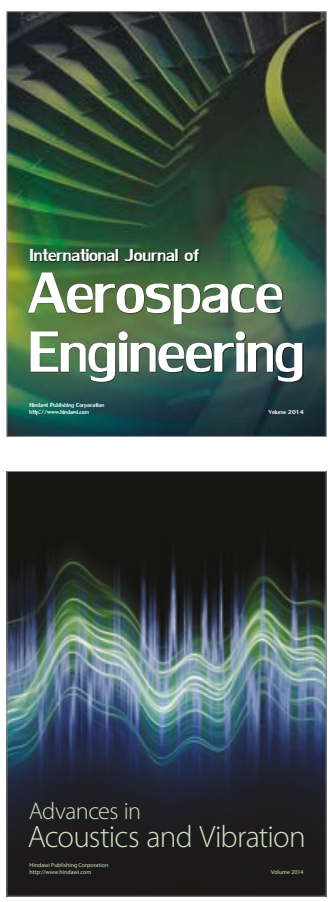

Sensor Networks 